\title{
Frações de fósforo e simbiose micorrízica em floresta secundária em resposta a disponibilidade de água e nutrientes na Amazônia oriental
}

\author{
Rodrigo da Silva MAIA ${ }^{1}$, Steel Silva VASCONCELOS ${ }^{2}$, Claudio José Reis de CARVALHO² \\ 1 Universidade Federal do Pará (UFPA), Instituto de Geocências, Programa de Pós-graduação em Ciências Ambientais, Rua Augusto Corrêa, 1, Belém, Pará, CEP 66075-110, Brasil \\ 2 Embrapa Amazônia Oriental, Laboratório de Análise de Sistemas Sustentáveis, Tv. Dr. Enéas Pinheiro, s/no, Belém, Pará, CEP 66095-100, Brasil. \\ * Autor correspondente: steel.vasconcelos@embrapa.br
}

\section{RESUMO}

A disponibilidade de nutrientes, especialmente fósforo, e de água no solo influenciam fortemente a simbiose micorrízica em ecossistemas florestais. O objetivo do estudo foi avaliar o impacto da remoção de serapilheira e do aumento da disponibilidade de água no solo sobre a densidade de esporos e a colonização micorrízica em raízes apogeotrópicas e não-apogeotrópicas em floresta secundária na Amazônia oriental. Foram analisadas a porcentagem de colonização micorrízica de raízes finas (diâmetro $\leq 2 \mathrm{~mm}$ ) apogeotrópicas e não-apogeotrópicas (presentes na camada de 0-10 cm do solo), a densidade de esporos, o teor de glomalina e a disponibilidade de fósforo no solo. A remoção de serapilheira não reduziu a disponibilidade de fósforo no solo. A densidade de esporos também não foi afetada pela remoção de serapilheira. De forma geral, as variáveis estudadas variaram sazonalmente, com exceção da colonização micorrízica em raízes não-apogeotrópicas e do teor de glomalina, mas não foram afetadas pela alteração na disponibilidade de água no solo decorrente da irrigação. Danos mecânicos ao sistema radicular apogeotrópico, inerentes à remoção quinzenal da serapilheira, devem ter contribuído para reduzir a colonização micorrízica em raízes apogeotrópicas e não-apogeotrópicas e, consequentemente, o teor de glomalina no solo. Os resultados deste estudo sugerem que a redução da cobertura do solo pode impactar negativamente a simbiose fungo-planta.

PALAVRAS-CHAVE: floresta sucessional, glomalina, umidade do solo

\section{Soil phosphorus fractions and mycorrhizal symbiosis in response to the availability of moisture and nutrients at a secondary forest in eastern Amazonia}

\begin{abstract}
The availability of soil nutrients (especially phosphorus) and soil water strongly influence mycorrhizal symbiosis in forest ecosystems. The aim of this study was to evaluate the impact of litter removal and increased soil moisture availability on the spore density and mycorrhizal colonization of apogeotropic and non-apogeotropic roots of a secondary forest stand in eastern Amazonia. We determined the percentage of mycorrhizal colonization of apogeotropic and non-apogeotropic $(0-10 \mathrm{~cm}$ soil depth) fine roots (diameter $\leq 2 \mathrm{~mm}$ ), spore density, glomalin concentration, and soil phosphorus availability. Litter removal did not reduce soil phosphorus availability. The spore density was not affected by litter removal. In general, the variables varied on a seasonal basis, except the mycorrizal colonization of non-apogetropic roots and glomalin concentration, but were not affected by irrigation. Mechanical damage to the apogeotropic root system, inherent to the fortnightly litter removal, may have contributed to decrease mycorrhizal colonization of both apogeotropic and non-apogeotropic roots and, consequently, soil glomalin. Our results suggest that the reduction of soil cover may have negative impact over the fungus-plant symbiosis.
\end{abstract}

KEYWORDS: glomalin, soil moisture, successional forest 


\section{INTRODUÇÃO}

A agricultura de derrubada e queima, tradicionalmente empregada por pequenos produtores na Amazônia, consiste na alternância de ciclos de cultivo e pousio (Homma 1998). Durante o pousio, um processo lento e gradativo de recuperaçáo da fertilidade do solo é determinado pelo crescimento da vegetaçáo secundária (Pereira e Vieira 2001), que promove importantes serviços ambientais ligados ao restabelecimento da ciclagem de carbono, nutrientes minerais e água (Nepstad et al. 2001).

A baixa disponibilidade de nutrientes, principalmente de fósforo, é comum nos solos amazônicos e pode limitar o crescimento da vegetação secundária (Davidson et al. 2004; Gehring et al. 1999). No entanto, florestas tropicais apresentam estratégias para maximizar a absorção de nutrientes, como associações com fungos micorrízicos e produção de raízes apogeotrópicas (Jordan 1982). Na Amazônia oriental, florestas secundárias geralmente conseguem se desenvolver em solos com elevada deficiência de fósforo (Davidson et al. 2004), possivelmente em parte devido ao estabelecimento de simbiose micorrízica.

Diversos fatores ambientais podem influenciar a colonização micorrízica e o crescimento radicular em ecossistemas florestais, tais como disponibilidade de nutrientes e água no solo, além de mudanças na cobertura do solo (Siqueira et al. 2007; Zangaro et al. 2009). Um experimento de longa duração de manipulação da disponibilidade de nutrientes e água, respectivamente por meio da remoção da serapilheira e irrigação, em floresta secundária localizada em Castanhal, Amazônia oriental, resultou em alteração de processos ligados ao ciclo de carbono e de nitrogênio na parte aérea das plantas e no solo (Fortini et al. 2003; Lima et al. 2010; Vasconcelos et al. 2008). No entanto, cinco anos consecutivos de remoção de serapilheira no mesmo experimento não reduziu o teor de fósforo na serapilheira produzida (litterfall), sugerindo que as plantas da vegetação secundária conseguiram manter a absorção desse nutriente, provavelmente em função da associação micorrízica (Vasconcelos et al. 2008). Uma melhor compreensão sobre as relaçóes entre micorrizas arbusculares, disponibilidade de água e mudanças na cobertura do solo deve ajudar no entendimento da resposta de florestas secundárias a perturbaçóes durante o processo sucessional, sobretudo aquelas perturbaçôes que influenciam a dinâmica de serapilheira, nutrientes e água.

Mudanças na cobertura do solo podem provocar impactos diretos sobre os fungos micorrízicos arbusculares (Brundrett et al. 1996) e alteraçôes na disponibilidade de água podem afetar a esporulação e a colonização micorrízica arbuscular (Augé 2004; Stutz e Morton 1996). Logo, foram levantadas as seguintes hipóteses principais: (a) a colonização micorrízica arbuscular aumentaria em função da redução da disponibilidade de nutrientes nas parcelas sob remoçáo de serapilheira e (b) a colonização micorrízica arbuscular seria alterada em função da aumento da disponibilidade de água nas parcelas irrigadas durante o período menos chuvoso. O objetivo do estudo foi avaliar o impacto da remoçáo de serapilheira e do aumento da disponibilidade de água no solo sobre a densidade de esporos, a colonizaçấo micorrízica em raízes apogeotrópicas e não-apogeotrópicas e a disponibilidade de fósforo em floresta secundária.

\section{MATERIAL E MÉTODOS}

\section{Área de estudo}

O estudo foi desenvolvido na área experimental do projeto Manflora (Manipulação da disponibilidade de água e nutrientes em floresta secundária na Amazônia oriental), localizada próximo ao $\mathrm{km} 63$ da rodovia BR 316, no distrito de Apeú, município de Castanhal, regiāo nordeste do Pará. Os solos da área de estudo são classificados como Latossolo Amarelo Distrófico Fase Pedregosa I (Concrecionário Laterítico) (Tenório et al. 1999). Na Tabela 1, são apresentadas algumas características químicas e físicas do solo. O clima é do tipo Am3, segundo a classificação de Köppen, com precipitaçấo pluvial média anual variando de 2.000 a 2.500 mm (Alvares et al. 2013).

Segundo os primeiros registros da área de estudo, a cobertura original era floresta tropical úmida, a qual, aproximadamente em 1939, foi derrubada para introduçăo de agricultura tradicional (derrubada-e-queima), envolvendo cultivos de milho (Zea mays L.) e mandioca (Manihot esculenta Crantz), com pousios de cerca de 10 anos (Aragão et al. 2005); a área foi abandonada pela última vez em 1987. Em inventário realizado em 2001, as quatro espécies arbóreas mais abundantes na área de estudo foram: Lacistema pubescens Mart. (6.700 indivíduos ha- ${ }^{-1}$, Myrcia sylvatica (G. Mey.) DC. (4300 indivíduos ha-1), Vismia guianensis Aubl. (933 indivíduos ha ${ }^{-1}$ ) e Cupania scrobiculata Rich. (892 indivíduos $\mathrm{ha}^{-1}$ ), representando $71 \%$ do total de indivíduos com diâmetro à altura do peito $\geq 1 \mathrm{~cm}$ (Araújo et al. 2005).

\section{Descrição dos experimentos}

A manipulação da disponibilidade de água e nutrientes foi realizada, respectivamente, por meio de um tratamento de irrigaçáo e de um tratamento de remoção de serapilheira, que foram comparados com o tratamento controle, em análises separadas. Dessa forma, o estudo consistiu em dois experimentos separados, i.e., experimento de irrigação durante o período menos chuvoso e experimento de remoção de serapilheira, embora as parcelas do tratamento controle tenham sido as mesmas para os dois experimentos. Foi utilizado um delineamento experimental inteiramente casualizado com quatro repetiçóes de cada tratamento. 
Tabela 1. Atributos químicos do solo (profundidade: 0-10 $\mathrm{cm}$ ) em parcelas controle, de irrigação e de remoção de serapilheira em floresta secundária na Amazônia oriental. Os dados são médias de amostragens realizadas nos períodos de coleta (chuvoso e menos chuvoso) $(n=8)$.

\begin{tabular}{lccc}
\hline & $\begin{array}{c}\text { Carbono orgânico } \\
\mathrm{mg} \mathrm{g}^{-1}\end{array}$ & $\begin{array}{c}\text { Nitrogênio total } \\
\mathrm{mg} \mathrm{kg}^{-1}\end{array}$ & $\mathrm{pH}$ \\
\hline Controle & 3,85 & 1,46 & 5,53 \\
Irrigação & 5,12 & 1,73 & 5,54 \\
Remoção & 2,11 & 1,16 & 5,52 \\
\hline
\end{tabular}

Em julho de 1999 foram instaladas 12 parcelas com dimensóes de $20 \mathrm{~m}$ x $20 \mathrm{~m}$ cada, sendo destinadas aleatoriamente quatro parcelas para o tratamento de irrigação, quatro parcelas para o tratamento de remoção de serapilheira e quatro parcelas para o tratamento controle. Parcelas adjacentes foram separadas por uma distância mínima de $10 \mathrm{~m}$. Cada parcela foi subdividida em quatro áreas, compostas cada uma de 12 subáreas para fins de amostragem de solo e raízes (Figura 1).

\section{Experimento de irrigação}

A irrigação foi implementada durante o período menos chuvoso (julho a dezembro), anualmente, de agosto de 2001 a dezembro de 2009. Com um sistema de irrigaçáo por microaspersão, foram aplicados $5 \mathrm{~mm}$ de água por dia, correspondentes às estimativas de evapotranspiração diária em florestas nativas da regiāo (Lean et al. 1996).

Experimento de remoçáo de serapilheira

A serapilheira foi removida manualmente, com auxílio de ancinho, a cada duas semanas, de agosto de 2001 a dezembro de 2009. Estima-se que tenham sido removidos $9,9 \mathrm{~kg} \mathrm{P} \mathrm{ha}^{-1}$ das parcelas de remoção de serapilheira entre agosto de 2001 e dezembro de 2004 (Vasconcelos et al. 2008).

\section{Coleta de solo e de raízes}

$\mathrm{Na}$ camada $0-10 \mathrm{~cm}$, foram coletadas, em cinco datas (fevereiro, abril, junho, agosto e setembro de 2009), amostras de solo para determinação de número de esporos, colonização micorrízica em raízes apogeotrópicas finas (diâmetro $\leq 2$ $\mathrm{mm}$ ) e umidade gravimétrica. Amostras para determinação de colonização micorrízica em raízes não-apogeotrópicas, teor de glomalina e teor de fósforo no solo foram coletadas em uma data no período chuvoso (abril de 2009) e outra no período menos chuvoso (setembro de 2009). Em cada parcela foi coletada aleatoriamente uma amostra em cada subárea em cada época de amostragem (Figura 1).

Em cada subárea, foram coletados cerca de $300 \mathrm{~g}$ de solo na camada 0-10 cm, $150 \mathrm{~g}$ de raízes apogeotrópicas finas e $150 \mathrm{~g}$ de raízes finas na camada $0-10 \mathrm{~cm}$ do solo. As amostras de solo foram coletadas com trado e usadas para análises de densidade de esporos e teor de glomalina total, assim como de teor de fósforo total, orgânico e disponível. As raízes apogeotrópicas foram coletadas manualmente na superfície do solo em uma área de aproximadamente $50 \mathrm{~cm}$ x $50 \mathrm{~cm}$. Raízes na camada $0-10 \mathrm{~cm}$ foram coletadas a partir da abertura de minitrincheiras com largura e comprimento de $10 \mathrm{~cm}$.

A extraçáo dos esporos (em amostras de $10 \mathrm{~g}$ de solo seco em ambiente natural) foi realizada com a técnica de peneiramento úmido (Gerdemann e Nicolson 1963), seguida de centrifugação em água e em sacarose a 45\% (Jenkins 1964), sendo quatro repetiçôes por amostra. A contagem do número de esporos foi realizada com auxílio de um microscópio estereoscópico (4x) (modelo DMW 143, Motic, Hong Kong, China). Apenas os esporos viáveis foram contados, isto é, aqueles que apresentaram suas estruturas preservadas.

Para avaliar a percentagem de colonizaçáo micorrízica (PCM), as raízes foram submetidas a um processo de clareamento e coloraçáo, de acordo com Phillips e Hayman (1970). Foram analisados no microscópio óptico 60 segmentos de raízes finas de $1 \mathrm{~cm}$ de comprimento por parcela. Para a contagem das interseçôes micorrízicas, utilizou-se o método da ampliação das interseçóes (McGonigle et al. 1990). Com o auxílio de uma ocular com escala milimetrada e uma objetiva de 10x, foram analisados segmentos de raiz a cada $1 \mathrm{~mm}$.

$\mathrm{O}$ teor de glomalina total foi determinado segundo Wright e Upadhyaya (1998). Em amostras de 0,5 g de solo foram adicionados $4 \mathrm{~mL}$ de citrato de sódio $50 \mathrm{mM}$, que foram submetidas à autoclavagem e, depois, centrifugação.

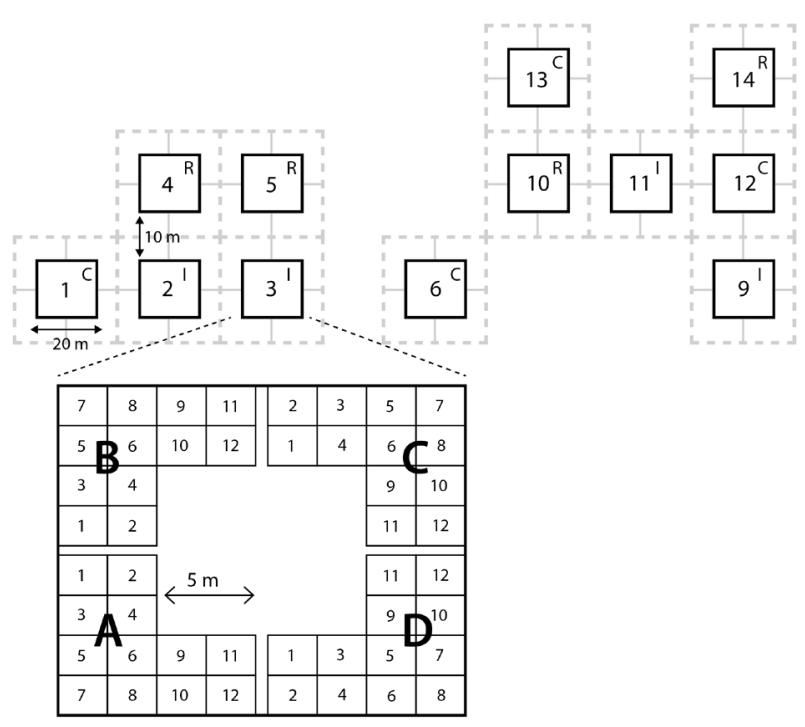

Figura 1. Croqui da área experimental, com detalhamento da delimitação de áreas $(A, B, C, D)$ e subáreas (1 a 12) usadas para a coleta de amostras de solo e raízes em floresta secundária na Amazônia oriental. As letras $C, R$ e I identificam os tratamentos controle, remoção de serapilheira e irrigação, respectivamente. 
Em seguida, foram adicionados imediatamente $5 \mathrm{~mL}$ do reativo de Bradford a uma alíquota de $100 \mu \mathrm{L}$ de amostra. A leitura foi realizada em comprimento de onda de 595 nm (espectrômetro UV/VIS, modelo 8675, UNICAM, Cambridge, Reino Unido).

A extração para determinaçáo do teor de fósforo total foi realizada de acordo com Nelson et al. (1953), utilizandose uma solução extratora de $2,0 \mathrm{~mL}$ de ácido sulfúrico concentrado em amostra de solo de aproximadamente $0,5 \mathrm{~g}$. Para a determinaçáo do teor de fósforo orgânico foi utilizada uma solução extratora de $25 \mathrm{~mL}$ de ácido sulfúrico $0,5 \mathrm{M} \mathrm{em}$ amostras de $1 \mathrm{~g}$ de solo previamente incineradas em mufla a $550{ }^{\circ} \mathrm{C}$ (Nelson et al. 1953). A determinação do teor de fósforo disponível foi realizado pelo método da adsorçáo em resina de troca aniônica, de acordo com Amer et al. (1955).

Os teores de fósforo total, orgânico e disponível foram determinados pelo método colorimétrico de Murphy e Riley (1962), em comprimento de onda de $885 \mathrm{~nm}$, usando-se um espectrômetro UV/VIS (modelo 8675, UNICAM, Cambridge, Reino Unido). O teor de fosforo orgânico foi obtido pela diferença entre o teor de fósforo na amostra não submetida à incineração e o teor de fósforo na amostra submetida à incineração.

\section{Análise estatística}

Análise de variância de medidas repetidas foi empregada para testar o efeito de tratamento, data de coleta e interação entre tratamento e data de coleta sobre as variáveis (colonização micorrízica, densidade de esporos, glomalina e fósforo do solo). As médias foram comparadas com o teste Tukey a $5 \%$. As análises foram realizadas com o programa estatístico Sigma Stat for Windows, Versão 3.5 (Systat Software. Inc., EUA, 2006).

\section{RESULTADOS}

\section{Experimento de remoção de serapilheira}

A umidade do solo foi afetada significativamente por tratamento, época e interaçáo tratamento $\mathrm{x}$ época de amostragem (Tabela 2). No tratamento controle, a umidade do solo durante os meses menos chuvosos (agosto e setembro de 2009) foi significativamente menor do que nos meses chuvosos (fevereiro, abril e junho de 2009) (Figura 2A). No tratamento com remoçáo de serapilheira, a umidade em setembro de 2009 foi significativamente menor do que nos outros meses (Figura 2B). Durante a época chuvosa, a umidade do solo foi significativamente menor nas parcelas com remoção de serapilheira em relação ao controle (Figura 2B).

Considerando os atributos relativos aos fungos micorrízicos arbusculares, houve efeito significativo da interação tratamento $\mathrm{x}$ época de amostragem somente sobre a colonização micorrízica em raízes não-apogeotrópicas (Tabela 2). As demais variáveis foram influenciadas pelos tratamentos (colonização micorrízica em raízes não-apogeotrópicas, glomalina) ou épocas de amostragem (esporos).

A colonização micorrízica em raízes apogeotrópicas foi significativamente maior no controle do que no tratamento com remoçáo de serapilheira (Figura 2C). A densidade de esporos na época menos chuvosa foi significativamente menor do que na época chuvosa (Figura 2D). Em raízes não-apogeotrópicas $(0-10 \mathrm{~cm})$, a colonização micorrízica não variou entre épocas de amostragem no tratamento controle, mas foi significativamente maior na época menos chuvosa no tratamento com remoção de serapilheira (Tabela 2). Em ambas as épocas de amostragem, a colonização micorrízica em raízes

Tabela 2. Valor $P$ das fontes de variação das variáveis analisadas nos experimentos de remoção de serapilheira e irrigação. Ug: umidade gravimétrica do solo, CMra: colonização micorrízica em raízes apogeotrópicas, CMrna: colonização micorrízica em raízes não-apogeotrópicas, Pt: fósforo total, Po: fósforo orgânico, Pd: fósforo disponível.

\begin{tabular}{|c|c|c|c|c|c|c|}
\hline \multirow{2}{*}{ Variável } & \multicolumn{3}{|c|}{ Remoção de serapilheira } & \multicolumn{3}{|c|}{ Irrigação } \\
\hline & Tratamento & Época & Tratamento x Época & Tratamento & Época & Tratamento x Época \\
\hline Ug & $P=0,002$ & $P=0,001$ & $P=0,031$ & $P=0,002$ & $P=0,001$ & $P=0,001$ \\
\hline Esporos & $P=0,524$ & $P<0,001$ & $P=0,438$ & $P=0,159$ & $P=0,006$ & $P=0,188$ \\
\hline CMra & $P=0,001$ & $P=0,377$ & $P=0,904$ & $P=0,122$ & $P=0,844$ & $P=0,453$ \\
\hline CMrna & $P=0,001$ & $P=0,003$ & $P=0,030$ & $P=0,542$ & $P=0,455$ & $P=0,642$ \\
\hline Glomalina & $P=0,022$ & $P=0,170$ & $P=0,554$ & $P=0,762$ & $P=0,285$ & $P=0,651$ \\
\hline Pt & $P=0,145$ & $P=0,093$ & $P=0,505$ & $P=0,268$ & $P=0,509$ & $P=0,384$ \\
\hline Po & $P=0,212$ & $P=0,003$ & $P=0,396$ & $P=0,355$ & $P=0,006$ & $P=0,649$ \\
\hline $\mathrm{Pd}$ & $P=0,823$ & $P=0,003$ & $P=0,953$ & $P=0,293$ & $P=0,005$ & $P=0,704$ \\
\hline
\end{tabular}


não-apogeotrópicas foi significativamente maior no controle (Tabela 3). O teor de glomalina total foi significativamente maior no controle. A disponibilidade de $\mathrm{P}$ total no solo não foi afetada pela remoção de serapilheira (Tabela 2, Figura 3A) e houve redução significativa nas fraçóes de $\mathrm{P}$ orgânico e $\mathrm{P}$ disponível (Figura 3B-C) durante a época menos chuvosa.

\section{Experimento de irrigação}

Houve efeito significativo da interação tratamento $\mathrm{x}$ época de amostragem sobre a umidade do solo (Tabela 2). Durante o período de irrigação, a umidade do solo nas parcelas irrigadas foi significativamente maior do que no controle (Figura 4B). A colonizaçáo micorrízica em raízes apogeotrópicas (Figura 4C) e
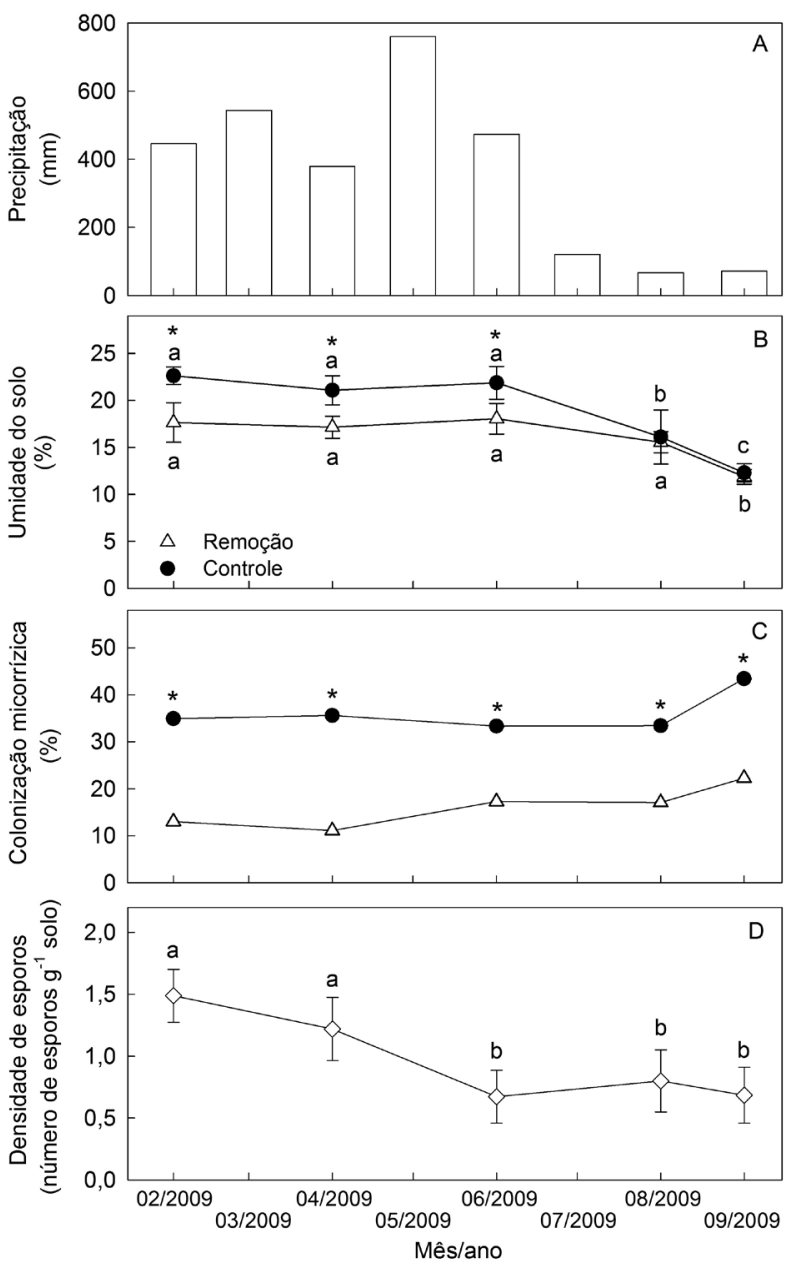

Figura 2. Precipitação pluvial mensal $(A)$ e efeito da remoção de serapilheira sobre a umidade do solo (B), colonização micorrízica em raízes apogeotrópicas (C) e densidade de esporos (D), em floresta secundária na Amazônia oriental. Nas Figuras B-C, os símbolos fechados e abertos representam as médias ( \pm desvio padrão) dos tratamentos controle e com remoção de serapilheira, respectivamente $(n=4)$; na Figura $D$, os símbolos representam a média geral dos tratamentos $(n=8)$. Astericos $\left.{ }^{*}\right)$ indicam diferença significativa entre tratamentos em uma data de coleta; letras indicam diferença significativa entre datas para um dado tratamento (teste Tukey, $\mathrm{P}<0,05$ ).
Tabela 3. Colonização micorrízica em raízes não-apogeotrópicas e teor de glomalina do solo em parcelas controle e de remoção de serapilheira em floresta secundária na Amazônia oriental. Dados: média \pm desvio padrão $(n=4)$.

\begin{tabular}{lccc}
\hline $\begin{array}{l}\text { Tratamento de } \\
\text { manipulação }\end{array}$ & $\begin{array}{c}\text { Período } \\
\text { chuvoso }\end{array}$ & $\begin{array}{c}\text { Período menos } \\
\text { chuvoso }\end{array}$ & $\begin{array}{c}\text { Média de } \\
\text { períodos }\end{array}$ \\
\hline Colonização micorrízica(\%)
\end{tabular}

Médias seguidas de letras iguais, maiúsculas nas linhas e minúsculas nas colunas, não diferem significativamente pelo teste Tukey $(P<0,05)$.

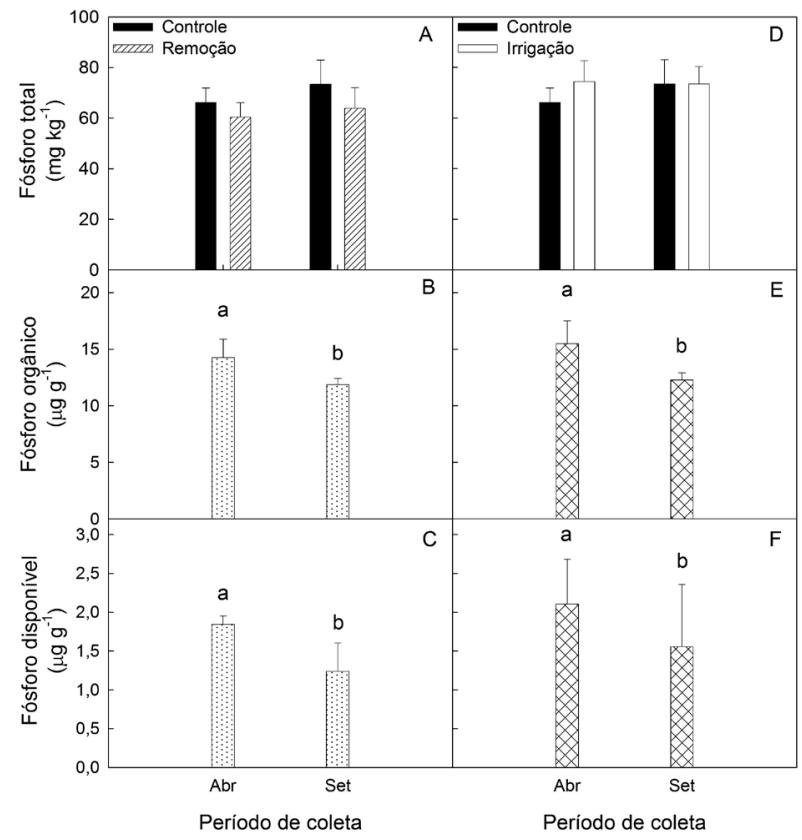

Figura 3. Variação temporal do teor total e de frações de fósforo do solo nos experimentos de irrigação e remoção de serapilheira em floresta secundária na Amazônia oriental. Nas Figuras A e D, as barras preta, branca e hachurada indicam as médias ( \pm desvio padrão) dos tratamentos controle, irrigação e remoção de serapilheira, respectivamente $(n=4)$. Nas Figuras B-C e E-F, as barras hachuradas representam as médias gerais $(n=8)$ dos tratamentos nos experimentos de remoção de serapilheira e irrigação, respectivamente (teste Tukey, $\mathrm{P}<0,05$ ). 
Tabela 4. Colonização micorrízica em raízes não-apogeotrópicas e teor de glomalina do solo em parcelas controle e de remoção de serapilheira em floresta secundária na Amazônia oriental. Dado: média \pm desvio padrão $(n=4)$.

\begin{tabular}{|c|c|c|c|c|}
\hline \multirow{2}{*}{$\begin{array}{l}\text { Tratamento de } \\
\text { manipulação }\end{array}$} & \multicolumn{2}{|c|}{ Colonização micorrízica (\%) } & \multicolumn{2}{|c|}{ Glomalina total (mg g-1) } \\
\hline & Período chuvoso & Período menos chuvoso & Período chuvoso & Período menos chuvoso \\
\hline Controle & $30,75 \pm 1,40$ & $33,81 \pm 0,04$ & $11,13 \pm 1,43$ & $11,94 \pm 1,35$ \\
\hline Irrigação & $30,37 \pm 0,70$ & $31,10 \pm 0,08$ & $11,60 \pm 1,43$ & $11,94 \pm 0,64$ \\
\hline
\end{tabular}
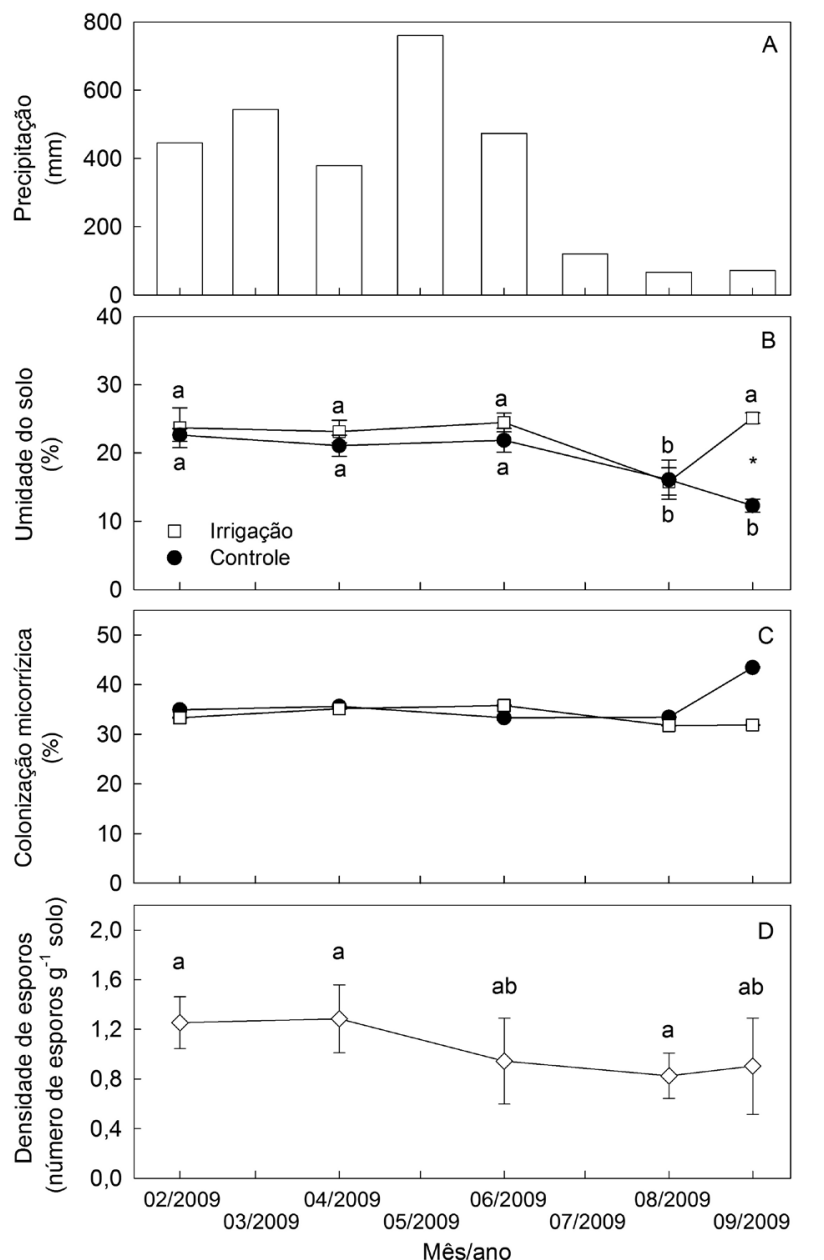

Figura 4. Precipitação pluvial mensal $(A)$ e efeito da irrigação durante a época menos chuvosa sobre a umidade do solo (B), colonização micorrízica em raízes apogeotrópicas (C) e densidade de esporos (D), em floresta secundária na Amazônia oriental. Nas Figuras B-C, os símbolos fechados e abertos representam as médias ( \pm desvio padrão) dos tratamentos controle e irrigação, respectivamente $(n=4)$; na Figura $D$, os símbolos representam a média geral dos tratamentos $(n=8)$. Asteriscos $\left(^{*}\right)$ indicam diferença significativa entre tratamentos em uma data de coleta; letras indicam diferença significativa entre datas para um dado tratamento (teste Tukey, $\mathrm{P}<0,05$ ). não-apogeotrópicas (Tabela 4), assim como o teor de glomalina total (Tabela 4), não foram sensíveis a alteraçôes na umidade do solo durante a irrigação na época menos chuvosa (Tabela 2). No entanto, houve tendência de maior colonização micorrízica na irrigação em setembro de 2009 (Figura 4C). No intervalo de mediçôes (fevereiro a setembro de 2009), a densidade de esporos variou temporalmente, com valor máximo na época chuvosa e valor mínimo na época menos chuvosa (Figura 4D).

A disponibilidade de fósforo total no solo (Figura 3D) apresentou tendência de ser maior no tratamento de irrigaçáo em abril, porém não houve diferença significativa. Os teores de fósforo orgânico e fósforo disponível foram afetados significativamente pela época de amostragem, com valores maiores na época chuvosa (Figura 3E-F).

\section{DISCUSSÃO}

\section{Efeitos da remoção de serapilheira}

A ausência de alteração na disponibilidade de $\mathrm{P}$ total do solo nas parcelas com remoção de serapilheira está de acordo com os resultados de outro experimento de remoçáo de serapilheira realizado em floresta tropical madura no Panamá (Sayer et al. 2012). Esses resultados sugerem que a serapilheira pouco influencia no fornecimento de $\mathrm{P}$ para o solo na área de estudo, provavelmente devido ao baixo teor de P da serapilheira (Vasconcelos et al. 2008). O retorno médio de $\mathrm{P}$ na serapilheira (litterfall) na área de estudo $(-3,0 \mathrm{~kg} P$ $\mathrm{ha}^{-1}$ ano $^{-1}$, Vasconcelos et al. 2008) é característico de solos de baixa fertilidade (Vitousek e Sanford 1986). A disponibilidade de fósforo no solo é quase exclusivamente controlada pelo material de origem do solo (Quesada et al. 2010). Fatores que contribuem para redução de $\mathrm{P}$ total no solo são atribuídos a perda de P orgânico e inorgânico dissolvido, diminuição da camada de proteção física no solo, processos de lixiviação ou perda de massa do solo durante o processo de intemperismo e obstrução permanente dos minerais do solo (Walker e Sayers 1976; Quesada et al. 2010). 
Em floresta tropical úmida no Panamá, após três anos de remoção de serapilheira, houve redução de $23 \%$ do teor de P orgânico nos primeiros $2 \mathrm{~cm}$ do solo (Vincent et al. 2010), correspondendo a uma perda de aproximadamente $1,4 \mathrm{~kg} \mathrm{ha}^{-1} \mathrm{ano}^{-1}$ de P. Considerando-se que nesta floresta no Panamá a demanda das plantas é cerca de $6,4 \mathrm{~kg} \mathrm{ha}^{-1}$ ano $^{-1} \mathrm{de}$ P (Kaspari et al. 2008), o P orgânico perdido pela remoção de serapilheira contribuiria com apenas $20 \%$ do total de P requerido para suprir o crescimento da floresta (Vincent $e t$ al. 2010), sugerindo que a maior proporção de P necessária para o crescimento das plantas deve ser obtida a partir do solo e náo necessariamente da decomposição da serapilheira. De fato, a produçáo de serapilheira fina, um dos principais componentes da produtividade primária liquida da parte aérea em ecossistemas florestais, não foi afetada pela remoção de serapilheira na mesma área experimental deste estudo (Vasconcelos et al. 2008).

Neste estudo, é possível que parte do P tenha sido suprido pela mineralização da matéria orgânica existente no solo antes do início da remoçáo da serapilheira, pois o teor de fraçôes de P-lábil pode ser alto em floresta secundária na Amazônia (Frizano et al. 2003; Markewitz et al. 2004). A elevada produção de fosfatase pelas raízes das plantas e microrganismos, comum em florestas secundárias com baixo teor de $\mathrm{P}$ (Marschner et al. 2002), é um mecanismo que disponibiliza o P retido em fraçóes lábeis (Oberson e Joner 2005).

Era esperado que a remoçáo da serapilheira reduzisse a disponibilidade de nutrientes do solo, ocasionando um déficit nutricional para a vegetaçáo secundária, o que estimularia o estabelecimento da simbiose com micorrizas arbusculares. De fato, a remoção da serapilheira afetou negativamente a ciclagem de $\mathrm{N}$, levando à redução no teor de $\mathrm{N}$ da serapilheira produzida (Vasconcelos et al. 2008). No entanto, o teor de $\mathrm{P}$ da serapilheira produzida não foi afetada pela remoção de serapilheira (Vasconcelos et al. 2008), consistente com a ausência de reduçáo do teor de P do solo neste estudo.

Diferentemente do resultado esperado, a colonizaçáo micorrízica diminuiu nas parcelas com remoçáo de serapilheira. Apesar da massa de raízes apogeotrópicas não ter sido quantificada neste estudo, o impacto causado pela remoção contínua da serapilheira visivelmente reduziu a extensão do sistema radicular apogeotrópico, que é o principal envolvido no estabelecimento da simbiose micorrízica na superfície do solo (Bellgard 1993; Brundrett 1991; Brundrett et al. 1996). Assim, danos ao sistema radicular apogeotrópico, resultantes do impacto mecânico da ferramenta utilizada no processo de remoçáo de serapilheira, muito provavelmente afetaram negativamente a colonização micorrízica.

As raízes finas nos primeiros centímetros do solo são provavelmente continuidade das raízes apogeotrópicas (Janos 1980). Logo, a redução de colonizaçáo micorrízica em raízes finas na camada $0-10 \mathrm{~cm}$ pode ser consequência da reduçáo da colonização em raízes apogeotrópicas. Esse resultado é consistente com estudos prévios na mesma área experimental que demonstraram redução na massa e no crescimento de raízes finas na profundidade de $0-10 \mathrm{~cm}$ do solo após cinco anos de remoção de serapilheira (Lima et al. 2010). A diminuição da colonização micorrízica consequentemente levou à redução no teor de glomalina, proteína produzida pelas hifas dos fungos micorrízicos arbusculares.

A remoção de serapilheira não alterou significativamente a densidade de esporos dos fungos micorrízicos arbusculares, possivelmente porque os esporos são resistentes a distúrbios ambientais (Brundrett 1991). Além disso, com exceção de algumas espécies como Glomus intraradices, que produz esporos no interior da raiz, a maioria dos fungos micorrízicos arbusculares produzem esporos diretamente no solo (Moreira e Siqueira 2006). Desse modo, os esporos foram menos prejudicados com a retirada de serapilheira do que as hifas e arbúsculos que ficam no interior das raízes.

A densidade de esporos foi afetada apenas no período menos chuvoso, embora os esporos sejam resistentes à seca (Johnson e Pfleger 1992). Dessa forma, presume-se que algumas espécies de fungos micorrízicos arbusculares são sensíveis a variaçóes de umidade no solo, como relatado por Tommerup (1984) e Sieverding (1983), reduzindo assim a produção de esporos durante o período menos chuvoso.

\section{Efeitos da sazonalidade da precipitação pluvial e da irrigação}

A redução da precipitação pluvial no período menos chuvoso refletiu em diminuição da umidade do solo nas parcelas controle. Com a irrigação, em setembro de 2009 a umidade do solo atingiu níveis próximos àqueles observados no período chuvoso. Alteraçóes no status hídrico do solo em função da sazonalidade da precipitação pluvial e da irrigação também foram demonstradas em estudos prévios na mesma área experimental (Fortini et al. 2003; Vasconcelos et al. 2008).

$\mathrm{O}$ aumento da umidade do solo durante o período menos chuvoso não foi suficiente para alterar significativamente a colonização micorrízica da vegetação secundária. Em um estudo prévio na mesma área de estudo, a massa e o comprimento de raízes finas na camada de $0-10 \mathrm{~cm}$ de solo também não sofreram alteração pela irrigação em relação ao controle (Lima et al. 2010). De modo geral, a colonização micorrízica é mais sensível em relação a variações extremas de umidade do solo (Cui e Nobel 1992; Stutz e Morton 1996), sugerindo que a diferença no status hídrico do solo entre parcelas irrigadas e controle não foi suficientemente intenso para afetar a colonização micorrízica. Geralmente o excesso de água no solo diminui a colonização micorrízica, enquanto 
que a seca crônica promove maior colonização de espécies vegetais cultivadas (Augé 2001).

A irrigação também não afetou a densidade de esporos, que aumentou apenas no período chuvoso em relação ao período menos chuvoso. No entanto, como os esporos são estruturas de resistência dos fungos micorrízicos arbusculares, apresentando elevada capacidade de sobrevivência sob condiçóes adversas, incluindo secas prolongadas e altas temperaturas (Brundrett 1991), esperava-se maior densidade de esporos no período menos chuvoso. A diminuição na densidade de esporos observada neste estudo, durante o período menos chuvoso, pode ser atribuída a algumas espécies de fungos micorrízicos arbusculares cuja produção de esporos é sensível a pequenas mudanças ambientais, como diminuição na umidade do solo. De fato, diversos autores (Tommerup 1984; Sieverding e Toro 1988; Sieverding 1983) encontraram variação na produção de esporos, dependendo da espécie de fungo micorrízico, sob diminuição da umidade do solo em casa de vegetação e campo.

Os teores de P orgânico e disponível foram maiores durante o período chuvoso em relaçáo ao período menos chuvoso. Quando a umidade do solo aumenta, o filme de água próximo às partículas do solo fica mais espesso, diminuindo a interação P-colóide (Tinker e Nye 2000). A forte interação entre P e colóides do solo reduz o transporte do elemento, especialmente em solos tropicais muito intemperizados, como é o caso dos solos amazônicos (Costa et al. 2006). Portanto, possivelmente o aumento do volume de água no solo durante o período chuvoso resultou em diminuição da interação entre P orgânico e colóide. A redução desta interação pode ter favorecido o transporte e disponibilização de P no solo (Costa et al. 2009). Além disso, o aumento da umidade do solo durante a época chuvosa pode favorecer a atividade decompositora dos microorganismos, aumentando a taxa de decomposição e disponibilização de nutrientes no solo (Luizão 2007).

\section{CONCLUSÕES}

A simbiose micorrízica não foi sensível ao aumento da umidade do solo decorrente da irrigação durante o período menos chuvoso. Não foi possível testar a hipótese de aumento da colonização micorrízica em funçáo do estresse nutricional nas parcelas de remoçáo de serapilheira, pois a concentração de fósforo do solo não diminuiu neste tratamento. Portanto, a redução da colonização micorrízica $(-30-50 \%)$ e do teor de glomalina $(-20 \%)$ nas parcelas de remoçáo de serapilheira devem ter resultado diretamente do impacto negativo do processo de remoção de serapilheira sobre o sistema radicular.

Os resultados deste estudo sugerem que a redução da cobertura do solo como, por exemplo, em função de desmatamento ou perturbaçóes abióticas, pode diminuir a simbiose micorrízica em florestas secundárias. Por outro lado, frações de fósforo no solo parecem não sofrer influência direta pela redução da cobertura vegetal, mas a disponibilidade de $\mathrm{P}$ orgânico e P disponível são sensíveis à variação da umidade solo.

\section{AGRADECIMENTOS}

Às equipes do Laboratório de Ecofisiologia e Propagação de Plantas da Embrapa Amazônia Oriental — Neusa Ferreira, Ivanildo Trindade e Cleo Marcelo de Araújo e da Estaçáo Experimental de Piscicultura de Água Doce - principalmente ao Sr. Raimundo Nonato e Paulo Alencar; aos comentários dos revisores, que resultaram na melhoria da qualidade do artigo; e à Coordenação de Aperfeiçoamento de Pessoal de Nível Superior (CAPES), pela bolsa de estudo, e ao Conselho Nacional de Desenvolvimento Científico e Tecnológico (CNPq), pelo financiamento da pesquisa.

\section{BIBLIOGRAFIA CITADA}

Alvares, C.A.; Stape, J.L.; Sentelhas, P.C.; Gonçalves, J.L.deM.; Sparovek, G. 2013. Köppen's climate classification map for Brazil. Meteorologische Zeitschrift, 22: 711-728.

Amer, F.; Bouldin, D.R.; Black, C.A.; Duke, F.R. 1955. Characterization of soil phosphorus by anion-exchange resin adsorption and $\mathrm{P}^{32}$-equilibration. Plant and Soil, 4: 391-408.

Aragão, D.V.; Fortini, L.B.; Mulkey, S.S.; Zarin, D.J.; Araujo, M.M.; Carvalho, C.J.R.de 2005. Correlation but no causation between leaf nitrogen and maximum assimilation: the role of drought and reproduction in gas exchange in an understory tropical plant Miconia ciliata (Melastomataceae). American Journal of Botany, 92: 456-461.

Araújo, M.M.; Tucker, J.M.; Vasconcelos, S.S.; Zarin, D.J.; Oliveira, W.; Sampaio, P.D.; et al. 2005. Padrão e processo sucessionais em florestas secundárias de diferentes idades na Amazônia oriental. Ciência Florestal, 15: 343-357.

Augé, R.M. 2001. Water relations, drought and vesicular arbuscular mycorrhizal symbiosis. Mycorrhiza, 11: 3-42.

Augé, R.M. 2004. Arbuscular mycorrhizae and soil/plant water relations. Canadian Journal of Soil Science, 84: 373-381.

Bellgard, S.E. 1993. The topsoil as the major store of propagules of vesicular arbuscular mycorrhizal fungi in southeast Australian sandstone soils. Mycorrhiza, 3: 19-24.

Brundrett, M.C. 1991. Mycorrhizas in natural ecosystems. Advances in Ecological Research, 21: 171-313.

Brundrett, M.C.; Ashwath, N.; Jasper, D.A. 1996. Mycorrhizas in the Kakadu region of tropical Australia: I. Propagules of mycorrhizal fungi and soil properties in natural habitats. Plant and Soil, 184: 159-171.

Costa, J.P.V.; Barros, N.F.; Albuquerque, A.W.; Filho, G.M.; Santos, J.R. 2006. Fluxo difusivo de fósforo em funçâo de doses e da umidade do solo. Revista Brasileira de Engenharia Agricola e Ambiental, 10: 828-835.

Costa, J.P.V.; Bastos, A.L.; Reis, L.S.; Martins, G.O.; Santos, A.F. 2009. Difusão de fósforo em solos de Alagoas influenciada por fontes do elemento e pela umidade. Revista Caatinga, 22: 229-235. 
Cui, M.; Nobel, P.S. 1992. Nutrient status, water uptake and gas exchange for three desert succulents infected with mycorrhizal fungi. New Phytologist, 122: 643-649.

Davidson, E.A.; Carvalho, C.J.R.; Vieira, I.C.G.; Figueiredo, R.O.; Moutinho, P.; Ishida, F.Y.; et al. 2004. Nitrogen and phosphorus limitation of biomass growth in a tropical secondary forest. Ecological Applications, 14: 150-163.

Fortini, L.B.; Mulkey, S.S.; Zarin, D.J.; Vasconcelos, S.S.; Carvalho, C.J.R.de 2003. Drought constraints on leaf gas exchange by Miconia ciliata (Melastomataceae) in the understory of an eastern Amazonian regrowth forest stand. American Journal of Botany, 90: 1064-1070.

Frizano, J.; Vann, D.R.; Johnson, A.H.; Johnson, C.M. 2003. Labile phosphorus in soils of forest fallows and primary forest in the Bragantina Region, Brazil. Biotropica, 35: 2-11.

Gehring, C.; Denich, M.; Kanashiro, M.; Vlek, P.L.G. 1999. Response of secondary vegetation in Eastern Amazonia to relaxed nutrient availability constraints. Biogeochemistry, 45: 223-241.

Gerdemann, J.W.; Nicolson, T.H. 1963. Spores of mycorrhizal Endogone species extracted from soil wet sieving and decanting. Transactions of the British Mycological Society, 46: 235-244.

Homma, A.K.O. 1998. Amazônia: Meio ambiente e desenvolvimento agrícola. 1. ed. Empresa Brasileira de Pesquisa Agropecuária, Brasília-DF, 412p.

Janos, D.P. 1980. Vesicular-arbuscular mycorrhizae affect lowland tropical rainforest plant growth. Ecology, 61:151-162.

Jenkins, W.R. 1964. A rapid centrifugal-flotation technique for separating nematodes from soil. Plant Disease Report, 48: 692.

Johnson, N.C.; Pfleger, F.L. 1992. Vesicular arbuscular mycorrhizae and cultural stresses. In: Bethlenfalvay, G.J.; Linderman, R.G. (Ed.) Mycorrhizae in sustainable agriculture. American Society of Agronomy, Madison, p.71-99.

Jordan, C. 1982. The nutrient balance of an Amazonian rain forest. Ecology, 63: 647-654.

Kaspari, M.; Garcia, M.N.; Harms, K.E.; Santana, M.; Wright, S.J.; Yavitt, J.B. 2008. Multiple nutrients limit litterfall and decomposition in a tropical forest. Ecology Letters, 11:35-43.

Lean, J.; Bunton, C.B.; Nobre, C.A.; Rowntree, P.R. 1996. The simulated impact of Amazonian deforestation on climate using measured ABRACOS vegetation characteristics. In: Gash, J.; Nobre, C.; Roberts, J.; Victoria, R. (Ed.) Amazonian deforestation and climate. John Wiley \& Sons, New York, p.549-576.

Lima, T.T.S.; Miranda, I.S.; Vasconcelos, S.S. 2010. Effects of water and nutrient availability on fine root growth in eastern Amazonian forest regrowth, Brazil. New Phytologist, 187: 622-630.

Luizão, F.J. 2007. Ciclos de nutrientes na Amazônia: respostas às mudanças ambientais e climáticas. Ciência e Cultura, 59: 31-36.

Markewitz, D.; Davidson, E.; Moutinho, P.; Nepstad, D. 2004. Nutrient loss and redistribution after forest clearing on a highly weathered soil in Amazonia. Ecological Applications, 14: 177-199.

Marschner, P.; Marino, W.; Lieberei, R. 2002. Seasonal effects on microorganisms in the rhizosphere of two tropical plants in a polyculture agroforestry system in Central Amazonia, Brazil. Biology and Fertility of Soils, 35: 68-71.
McGonigle, T.P.; Miller, M.H.; Evans, D.G.; Fairchild, G.L.; Swan, J.A. 1990. A new method which gives an objective measure of colonization of roots by vesicular-arbuscular mycorrhizal fungi. New Phytologist, 115: 495-501.

Moreira, F.M.S.; Siqueira, J.O. 2006. Microbiologia e bioquimica do solo. 2 da ed., Universidade Federal de Lavras, Lavras, 729p.

Murphy, J.; Riley, J.P. 1962. A modified single solution method for the determination of phosphate in natural waters. Analytica Chimica Acta, 27: 31-36.

Nelson, W.L.; Mehlich, A.; Winters, E. 1953. The development, evaluation and use of soil tests for phosphorus availability. Agronomy, 14: 153-188.

Nepstad, D.; Moutinho, P.R.S.; Markewitz, D. 2001. The recovery of biomass, nutrient stocks, and deep-soil functions in secondary forests. In: McClain, M.E.; Victoria, R.L; Richey, J.E. (Ed.) The biogeochemistry of the Amazon Basin. University Press, Oxford, p.139-155.

Oberson, A; Joner, E.J. 2005. Microbial turnover of phosphorus in soil. In: Turner, B.L.; Frossard, E.; Baldwin, D.S. (Ed.) Organic phosphorus in the environment. CABI Publishing, Oxford, p.133-164.

Pereira, C.A; Vieira, I.C.G. 2001. A importância das florestas secundárias e os impactos de sua substituição por plantios mecanizados de grãos na Amazônia. Interciência, 26: 337-340.

Phillips, J.M.; Hayman, D.S. 1970. Improved procedures for clearing roots and staining parasitic and vesicular-arbuscular mycorrhizal fungi for rapid assessment of infection. Transactions of the British Mycological Society, 55: 158-161.

Quesada, C.A.; Lloyd, J.; Schwarz, M.; Patiño, S.; Baker, T.R.; Czimczik, C.; et al. 2010. Variations in chemical and physical properties of Amazon forest soils in relation to their genesis. Biogeosciences, 7: 1515-1541.

Sayer, E.J.; Wright, S.J.; Tanner, E.V.J.; Yavitt, J.B.; Harms, K.E.; Powers, J.S.; et al. 2012. Variable responses of lowland tropical forest nutrient status to fertilization and litter manipulation. Ecosystems, 15: 387-400.

Sieverding, E. 1983. Influence of soil water regimes on VA mycorrhiza. II. Effect of soil temperature and water regime on growth, nutrient uptake, and water utilization of Eupatorium odoratum L. Journal of Agronomy and Crop Science, 152: 56-67.

Sieverding, E.; Toro, T.S. 1988. Influence of soil water regimes on VA mycorrhiza. V. Performance of different VAM fungal species with cassava. Journal of Agronomy and Crop Science, 161: 322-332.

Siqueira, J.O.; Soares, C.R.F.S.; Santos, J.G.D.dos; Schneider, J.; Carneiro, M.A.C. 2007. Micorrizas e degradação do solo: Caracterização, efeitos e ação recuperadora. In: Tópicos em ciência do solo. v.5, Sociedade Brasileira de Ciência do Solo, Viçosa, p.219-256.

Stutz, J.C.; Morton, J.B. 1996. Successive pot cultures reveal high species richness of arbuscular endomycorrhizal fungi in arid ecosystems. Canadian Journal of Botany, 74: 1883-1889.

Tenório, A.R.M.; Graça, J.J.C.; Góes, J.E.M.; Mendes, J.G.R.; Gama, J.R.N.F.; Silva, P.R.O.; et al. 1999. Mapeamento dos solos 
da Estação de Piscicultura de Castanhal. 1ra ed., Faculdade de Ciências Agrárias do Pará, Belém, p.1-27.

Tinker, P.B.; Nye, P.H. 2000. Solute movement in the rhizosphere. Oxford University Press, Oxford, 444p.

Tommerup, I.C. 1984. Effect of soil water potential on spore germination by vesicular-arbuscular mycorrhizal fungi. Transactions of the British Mycological Society, 83: 193-202.

Vasconcelos, S.S.; Zarin, D.J.; Araújo, M.M.; Rangel-Vasconcelos, L.G.T.; Carvalho, C.J.R.; Staudhammer, C.L.; et al. 2008. Effects of seasonality, litter removal and dry-season irrigation on litterfall quantity and quality in eastern Amazonian forest regrowth, Brazil. Journal of Tropical Ecology, 24:27-38.

Vincent, A.G.; Turner, B.L.; Tanner, E.V.J. 2010. Soil organic phosphorus dynamics following perturbation of litter cycling in a tropical moist forest. European Journal of Soil Science, 61: 48-57.
Vitousek, P.M.; Sanford, R.L. 1986. Nutrient cycling in moist tropical forest. Annual Review of Ecology and Systematics, 17: 137-167.

Walker, T. W.; Syers, J. K. 1976. The fate of phosphorus during pedogenesis. Geoderma, 15: 1-19.

Wright, S.F; Upadhyaya, A. 1998. A survey of soils for aggregate stability and glomalin, a glycoprotein produced by hyphae of arbuscular mycorrhizal fungi. Plant and Soil, 198: 97-107.

Zangaro, W.; Nogueira, M.A.; Andrade, G. 2009. Arbuscular mycorrhizal fungi used as biofertilizers in revegetation programmes. In: Rai, M. (Org.). Advances in fungal biotechnology. 1st. ed., International Publishing House, New Delhi, p.351-378.

Recebido em 29/07/2014

Aceito em 20/02/2015 